ARTÍCULO ORIGINAL

\title{
Asociatividad en las mujeres tejedoras paraguayas en el distrito de Yataity, Guairá, Paraguay
}

\author{
Associativity in Paraguayan women weavers in the district of Yataity, Guaira, \\ Paraguay
}

\author{
Selva Margarita Olmedo Barchello ${ }^{1}$, Marcela Fernanda Achinelli Báez ${ }^{1}$, Dahiana \\ Elizabeth Ayala Alfonso ${ }^{1}$
}

\begin{abstract}
Resumen: El presente trabajo tiene como objetivo recopilar la experiencia de asociatividad en mujeres tejedoras paraguayas en el distrito de Yataity, Departamento de Guaira; considerada cuna del ao po'i (tejido tradicional). El trabajo de artesanía está caracterizado por ser un oficio familiar que se transmite de generación en generación. La producción y la comercialización de bienes culturales no solo puede considerarse una alternativa para que las mujeres puedan obtener ingresos y lograr la autonomía económica, sino que trae consigo innumerables beneficios para la familia y la comunidad además de generar procesos de asociatividad entre las mujeres. El desarrollo de la investigación se realizó a través de un análisis documentalbibliográfico, recopilando datos estadísticos de la Dirección General de Estadística, Encuestas y Censos y el Instituto Paraguayo de Artesanía, además de la aplicación de grupos focales con los distintos comités de artesanas tejedoras del Departamento de Guaira. La realidad encontrada es que este sector se encuentra vulnerable y sin la debida aplicación de politicas públicas, sin el cual no se lograría la reducción de la condición de pobreza de muchas mujeres artesanas y el ansiado camino hacia el desarrollo económico y social; donde el sector cooperativo tiene un papel clave como promotor de los principios de igualdad, equidad y solidaridad.
\end{abstract}

Palabras clave: Empoderamiento, mujeres, artesania, cooperativas de trabajo, asociatividad, cultura.

Abstract: This work aims to gather the experience of associativity Paraguayan women weavers in the district of Yataity, Guaira; po'i considered the cradle of the year. The craftsmanship is characterized as a trade that is passed from generation to generation family. Production and marketing of cultural goods not only be considered an alternative for women to earn income and achieve economic autonomy, but brings many benefits to the family and the community and generate processes associativity among women. The development of the research conducted through a documentary-bibliographical analysis, compiling statistics of the Directorate General of Statistics, Surveys and Censuses and the Paraguayan Institute of Handicrafts, besides the application of focus groups with the various committees of artisan weavers Guaira. The reality is found that this sector is vulnerable without the proper implementation of public policies, without which the reduction of poverty status of many women artisans and the long-awaited road to economic and social development would not be achieved; where the cooperative sector has a key role in promoting the principles of equality, equity and solidarity.

Keywords: Empowerment, women, crafts, work cooperatives, associations, culture.

1 Facultad de Ciencias Económicas, Universidad Nacional de Asunción. San Lorenzo, Paraguay. (selvaob@gmail.com), (machinellib@gmail.com), (dahianaayala288@gmail.com).

Recibido: 31/03/2016; Aceptado: 30/06/2016.

http:/ /dx.doi.org/ 10.18004/riics.2016.julio.43-60

ISSN (Impresa) 2225-5117. ISSN (En Línea) 2226-4000.

Rev. Int. Investig. Cienc. Soc. Vol. $12 \mathrm{n}^{\circ} 1$, julio 2016. pág. 43-60. 


\section{INTRODUCCIÓN}

Coque Martinez señala que la perspectiva histórica de América Latina se remonta a las formas de vivir en comunidad existente antes de la llegada de los europeos a tierras americanas (Martínez, 2002).

Los conquistadores españoles han encontrado una portentosa abundancia de productos agrícolas en las tierras guaraníes, lo cual está documentado en el escrito del gobernador Domingo Martínez de Irala en 1541, que "se tiene tanta abundancia de mantenimientos, que no sólo hay para la gente que alli reside, más para más de otros tres mil hombres encima". Para el guaraní, la tierra nunca fue un simple medio de producción económica, sino, ante todo, un espacio sociopolítico.

"El tekoha significa y produce al mismo tiempo relaciones económicas, sociales y organización político-religiosa, esenciales para la vida guaraní". El tekoha es una interrelación de espacios físico-sociales. Según Bartomeu Meliá, el lugar de pesca y caza, cultivable y de la casa, son los tres espacios que definen la bondad de la tierra guaraní. La economía y sociedad se muestran indisolublemente relacionadas en estos tres aspectos, que a su vez representan símbolos religiosos, porque reflejan la experiencia de Dios, ya que la tierra es el fundamento esencial de la concepción religiosa del guaraní. Gracias a la tierra y viviendo en ella, se logra la perfección de la vida económica y sociopolítica (Bremer, 2016).

La tierra es el fundamento para su economía de reciprocidad. Un consejo guaraní dice:

Habiendo conseguido la plenitud de tus frutos, darás de comer de ellos a todos tus vecinos sin excepción. Los frutos perfectos se producen para que de ellos coman todos, y no para que sean objetos de tacañeria. Dando de comer a todos, sólo así, viendo nuestro Padre nuestro amor a todos, alargará nuestros días para que podamos sembrar repetidas veces (Bremer, 2016).

Para la economía guaraní es esencial el trabajo en común, el potiró. Es una ayuda mutua y gratuita. Esto hoy se llamaria, en la sociedad moderna "solidaridad". El proceso de trabajo y de producción está, para el guaraní, no sólo condicionado sino hasta determinado a reproducir el don; es decir, tiene la reciprocidad, el jopói, su razón práctica económica. Convite y fiesta "son primero y último producto de esta economía de trabajo" dice Meliá. En el potiró y el jopói el 
“modo de ser guarani”" se hace realidad. Queda claro que la economía de reciprocidad no es un sistema de intercambio; no se basa en el interés para sí, sino en el interés para el otro, es el gesto del don. Dice Meliá: "el respeto al Otro, el reconocimiento del Otro hace descubrir no solamente al otro en sí mismo, sino eso nuevo, que entre el otro y si mismo, nace en cuanto humanidad. Y porque no está dentro de las cosas dadas y recibidas, y menos aún en las cosas intercambiadas, ese valor es el que constituye toda la diferencia entre la economía de intercambio y la economía de reciprocidad". Esta economía de reciprocidad ha creado la idea de la tierra sin mal (Villamil Peña \& Quevedo, 1987).

\section{La herencia de la mujer paraguaya}

El corazón de América, como se ha denominado a Paraguay, tiene una larga historia de resistencia y gran parte de la construcción de la nación tiene como principal protagonista a la mujer paraguaya. Desde la conquista de América se destaca la resistencia de las mujeres indígenas a ser explotadas sexualmente por los colonizadores, el rol tan importante de las mujeres en la gesta independentista, recordar a las residentas, mujeres que sembraron la tierra, hilaron el algodón a medida que la Guerra de la Triple Alianza se desarrollaba y el ejército aliado avanzaba hacia el territorio nacional, ellas, al término de la guerra y con un país totalmente devastado se hicieron cargo de reconstruir la cultura, la identidad y el uso del idioma guaraní, asumiendo tareas productivas y de comercialización y se encargaban de gran parte de la economía de subsistencia, desde la mandioca, el tabaco y la caña de azúcar (Villamil Peña \& Quevedo, 1987).

Los guaraníes tenían los roles bien determinados, en términos feministas se puede redefinir como un acuerdo de género complementario similar a los demás pueblos originarios de América Latina (Espinosa Miñoso, Gómez Correal, \& Ochoa Muñoz, 2014), contrario al acuerdo de género europeo que ha considerado a los roles de género como opuestos (Beauvoir, López Pardina, \& Martorell, 2005; Espinosa Miñoso et al., 2014; Potthast, 1996). Las guaranies se encargaban de la economía de subsistencia y del comercio, siendo los hombres dedicados al rol politico y exportador (Potthast, 1996).

En la conquista, el rol de la india fue protagónico para la formación de la raza paraguaya, debido a que para la cultura guaraní, las mujeres eran consideradas el bien más importante de la sociedad, siendo las relaciones de parentesco la garantía para una alianza de familias; era considerado el sello de estabilidad. Potthast, citando a González en Proceso y Formación de la cultura 
paraguaya cita: "La alianza del indio y del europeo realizó la sintesis racial en el mestizo, primera expresión de una nueva humanidad... mediante este proceso, América comenzaba a conquistar al conquistador" (Potthast, 2006).

De ahí el hecho de que el cacique sea considerado como el hombre más importante de una comunidad, pues ellos poseen varias mujeres, es decir, varias alianzas de familias que se traduce en estabilidad política, económica y social (Potthast, 1996).

La guaraní fue la promotora de la cultura, pese a los diversos enfrentamientos internos existentes en época de la colonia, los cuales buscaron dilapidar cualquier rastro de la raza autóctona separando familias y ubicando al indio como último en el escalafón social; vio la forma de transmitir la raza, encontrando la manera a través del idioma (Potthast, 1996). La india ha sabido transmitir su resistencia, compañerismo, trabajo y vida en comunidad a sus hijos e hijas, siendo la fortaleza de las mujeres en la guerra guasu (Potthast, 2006).

En los gobiernos anteriores a la Guerra de la Triple Alianza, la mayoría de los hombres se dedicaba a la exportación de yerba, ganado y madera; en cambio las mujeres trabajaban en la economía de subsistencia. Esta manera de trabajar ayudo a que mucho tiempo después, la mujer paraguaya haga frente a la economía y al comercio, al igual que sus antepasadas (Potthast, 2006).

Ya en tiempos de pos-guerra, el exceso de población femenina, ha propiciado el mote de "País de las mujeres"; caracterizando a esta transición como el doble rol que han cumplido estas mujeres que han levantado a la patria. Potthast, cita a Rush en Die Paraguayer:

La mujer gobierna en el pueblo paraguayo de las capas bajas... son todas amazonas, que actúan con libertad e inteligencia masculina, que fuman sus cigarros fuertes de tipo "Brissago" y dan órdenes a los hombres. No hay que olvidar que esta clase de mujeres robustas y laboriosas, sin cuyo régimen enérgico los paraguayos seguirian siendo un pueblo bastante abandonado, surgió de los terribles años sesenta y setenta del siglo pasado, cuando toda la población masculina del país prácticamente estaba aniquilada... el hombre no vale nada en la familia, nada en los negocios, la mujer sólo le permite actuar como ciudadano, discutiendo los asuntos del país, y la política es la única ocupación del paraguayo que lo vuelve impulsivo y apasionado. Incluso parece que estas mujeres 
extrañas no quieren otra cosa, prefieren que sus hombres sean algo cómodos e incluso bastante reservados frente al trabajo. Parece que ellas se sienten más seguras en su rol como hombres" (Potthast, 1996).

A pesar de que las mujeres reconstruyeron la nación después de la Guerra de la Triple Alianza y que desde entonces muchas de ellas lucharon por derechos civiles y políticos, fue recién en el año 1961 que se aprobó el voto para las mujeres en el Paraguay.

\section{Asociatividad, género y empoderamiento}

Para Moser (1992) fueron las mujeres, a través del trabajo informal quienes han dado una respuesta empírica de sobrevivencia a la crisis global. (Serafini Geoghegan, 2008) Serafini, citando a Montaño señala que las mujeres de la región presentan como características comunes a las mujeres de la región latinoamericana, los empleos precarios, mayor jefatura femenina, brecha salarial $20 \%$ menor que la de los hombres, así como la condición de gratuidad de los servicios de cuidado, a pesar de que la escolaridad femenina superaba a la de los hombres.

En Paraguay la situación ha seguido la misma tendencia, acentuando que la organización complementaria de los roles de género trazada desde los guaraníes, destinando el rol del hombre a "lo público" y el de la mujer a "lo económico"; ha formado el sistema económico paraguayo (Potthast, 1996).

El episodio más importante de la historia paraguaya cita Potthast mencionando a Riveiro Da Silva, fue la reconstrucción de Paraguay tras la Guerra de la Triple Alianza, ha creado no solo desequilibrios demográficos, sino también ha dado una re-definición de la familia paraguaya; habiendo un hombre para muchas mujeres (Potthast, 1996).

Esta característica antropológica ha creado una idiosincrasia en la mujer paraguaya que la ha hecho enfrentarse a varias circunstancias contando más con la asociación de las pares que de sus maridos, esposos o amantes; la asociatividad en el trabajo de las mujeres se ha reproducido con mayor facilidad por dos factores fundamentales: la posibilidad de fusionar trabajo no remunerado y remunerado, es decir el cuidado de los hijos e hijas, sumado a alguna actividad que las retribuya económicamente. 
El empoderamiento económico de las mujeres ha existido siempre, sin embargo; lo que ha ido profundizando es la desigualdad de género, una deuda de la sociedad frente a las mujeres, otorgarle el reconocimiento, la justicia social. Si bien el trabajo ha estado presente, los derechos de la propiedad y la educación no han ido de la mano; por lo cual no podemos hablar de un empoderamiento real, sino más bien que podemos pensar en empoderamiento paliativo, a época de crisis y subsistencia (Sen \& Rabasco, 2000).

La región latinoamericana es el continente más desigual, la distribución de la riqueza beneficia al 10\% de los individuos, recibiendo el 50\% de las ganancias; mientras el $20 \%$ no es propietario ni del $5 \%$, caracterizado por una feminización de la pobreza (Espinosa Miñoso et al., 2014). Además, se profundiza la desigualdad desde el punto de vista territorial, siendo las zonas rurales, las más marginadas y sobre todo si su población corresponde a pueblos originarios o afro descendientes (Ranaboldo y Leiva, 2013). Esto se observa, a través de los indicadores de cobertura de servicios en salud y educación, factores que limitan las oportunidades económicas de las poblaciones rurales y en especial el de las mujeres. A todo esto, se suma el efecto de la globalización en la desigualdad, lo cual ha ahondado la brecha entre hombres y mujeres (Girón \& Vargas, 2009).

Paraguay tiene una población total de 7 millones de habitantes, siendo el $51 \%$ mujeres y el 49\% hombres. El 66\% representa la población de 18 y más años. En cuanto a la pobreza, se presentó niveles de reducción desde el año 2011, pasando de 32,4\% en el año 2011 y 22,6\% en el año 2014. Sin embargo, la pobreza sigue afectando a 1.530.381 personas; donde la pobreza extrema en el 2014 afectó a 710.173 personas.

Ahora bien, la fuerza de trabajo está compuesta por personas ocupadas y desocupadas, siendo el $87 \%$ hombres y $58 \%$ mujeres. Esto evidencia una gran cantidad de mujeres inactivas. La inactividad económica relaciona el fenómeno de la pobreza que afecta en mayor medida a las mujeres. Del total de hombres fuera de la fuerza laboral, el $80,6 \%$ es no pobre y el $19,4 \%$ es pobre, en el caso de las mujeres; el $78,0 \%$ es no pobre y el $22,0 \%$ es pobre, revelando así, que son las mujeres inactivas quienes se encuentran más desfavorecidas frente a los hombres.

Sin embargo, la oferta de trabajo para las mujeres ha venido creciendo en las últimas décadas, las mujeres urbanas muestran mayor disposición a trabajar en el mercado laboral frente a las mujeres rurales, esto puede deberse a factores sociales y culturales, como el mayor nivel educativo, la cobertura más ampliada de los 
servicios de cuidado y las mayores probabilidades de conseguir empleo en el área urbana.

Un análisis de la Encuesta Permanente de Hogares de Paraguay en el año 2008, realizado por Echauri y Serafini, sostiene que el 55,8\% de mujeres del sector urbano y el $83,1 \%$ de mujeres del sector rural concentraban sus actividades en tres categorias: cuenta propista, familiar no remunerado y empleada doméstica, frente al $25,7 \%$ y $63,1 \%$ de hombres, respectivamente. El análisis por área de residencia, arrojó que 5 de cada 10 trabajadoras estaba autoempleada. En la zona urbana, las mujeres que trabajaban por cuenta propia y en el trabajo doméstico remunerado fue de 19,5\% (Echauri y Serafini, 2011).

La exclusión laboral de las mujeres y la precariedad laboral a la que se enfrentan, además de constituirse en un obstáculo para su desarrollo personal, actúa como limitante en cuanto a la contribución de las mujeres a la reducción de la pobreza.

La invisibilidad de las labores de cuidado que son casi exclusivamente trabajo femenino, imposibilita la autonomía económica, además de pautas culturales y sociales que alientan a la exclusión económica de las mujeres.

La ampliación de las oportunidades económicas constituyen un pilar fundamental para lograr la autonomía económica de las mujeres y a través de ello la lucha contra la pobreza, la generación de ingresos es un camino importante pero ello debe ir acompañado de condiciones adecuadas que se generen por parte del estado, haciendo que las personas puedan pensar una vida digna reduciendo la incertidumbre y garantizando su bienestar y la de sus familias.

\section{Un trabajo cultural y económico: la confección del ao po’i}

El trabajo artesanal se encuentra en países desarrollados como en vías de desarrollo (UNESCO, 2009). Una característica de este tipo de trabajo, denominado muchas veces "el oficio", es el que se transmite de generación en generación familiar (Norverto, 2014), realizado mayoritariamente por la población femenina, lo cual añade otra característica ya que posibilita desarrollarse paralelamente a cualquier otra actividad (Forstner, 2013).

En Latinoamérica este saber hacer, esta transmisión cultural, es al igual que el lenguaje, un aprendizaje que se da per se, sea de pueblos originarios o no. Para 
las mujeres que viven en las zonas rurales las actividades rentables tienen un dificil acceso, por lo cual el trabajo de artesanía adquiere una dimensión importante (Ranaboldo \& Leiva, 2013). A partir de los años noventa, han crecido en los países latinoamericanos los ingresos y empleos rurales no agrícolas (Berdegue et al., 2014; Readon, 2001). La Comisión Económica para América Latina (CEPAL) en el año 2000, estimaba que 9 de 11 países entre el 65 y el 93\% de las mujeres que vivian en zonas rurales participaban en actividades no-agrícolas.

En Paraguay la institución encargada del fomento en cuanto a la producción y consumo de artesanía es el Instituto Paraguayo de Artesanía (IPA). Según el registro proveído por el IPA, existen 2.323 productores de bienes culturales (tejidos y bordados tradicionales), donde el $71,7 \%$ produce prendas en Ao Po'i, el $18 \%$ produce el bordado en Nandutí y el 10,3\% produce bordado en Algodón o Encaje Ju.

El Ao Po’i se produce en las ciudades de Villeta, Carapeguá, Pirayú, Asunción y el mayor exponente es el departamento de Guairá (que cuenta con varios distritos dedicados a este sector, entre ellos a Yataity, considerado cuna del ao po'i). En términos absolutos 1.666 artesanos trabajan el rubro de Ao Po’i, el $97,7 \%$ son mujeres y el $2,3 \%$ son hombres. Del total de productores de prendas de Ao Po'i, el 85,4\% se concentra en el departamento de Guairá, el 12,2\% en Central y el $2,5 \%$ en Cordillera.

\section{Las mujeres en las cooperativas de trabajo en Paraguay}

Paraguay cuenta con el segundo movimiento cooperativo de mayor importancia a nivel mundial, si se tiene en cuenta el gran impacto que tiene el sector en la economía, solamente superado por Alemania, los datos y los indicadores muestran un comportamiento creciente en un periodo de cuatro décadas, el número de cooperativas creció 15,5 veces (CONPACOOP, 2012), representando el número de socios el $20 \%$ de la población total aproximadamente.

El sector cooperativo paraguayo se ha consolidado después de la crisis financiera del año 1995; y luego del año 2000 se visualizan otras formas de cooperativismo, entre las cuales se encuentran las cooperativas de trabajo. Las cooperativas especializadas de trabajo, de acuerdo a su definición se constituyen con el fin de dar empleo a sus asociados; las personas se asocian para prestar algún servicio, producir bienes o ejecutar obras. Por definición, es la entidad la que 
busca opciones de trabajo para todos los socios, de manera colectiva no individual (Carosini \& Achinelli, 2015).

Para las mujeres, el sector cooperativo ha propiciado a través del órgano internacional de Alianza Internacional Cooperativa (ACI-América), principios de igualdad y equidad de las mujeres. Paraguay, a través de la Confederación Paraguaya de Cooperativas (CONPACOOP) integra a su estructura orgánica al Comité Nacional de Mujeres Cooperativistas como parte del proceso de inclusión de la perspectiva de género.

Si bien en el país existen antecedentes de intentos de formación de cooperativas de trabajo en el rubro artesanal, sin embargo no han sido favorables los resultados de conformación. Desde el año 2012 la CONPACOOP ha desarrollado un proyecto de promoción de cooperativas de trabajo asociado en la economía social y solidaria en Paraguay, el cual fue financiado por la Agencia Andaluza de Cooperación Internacional al Desarrollo (AACID) y ejecutado por la Escuela Andaluza de Economía Social (EAES) y la Cooperación para el Desarrollo de los Países Emergentes (COSPE, por sus siglas en italiano). En el mencionado proyecto formaron parte mujeres y varones de diferentes tipos de Cooperativas y PreCooperativas dedicadas a diversas actividades.

Un caso particular fue el de las artesanas tejedoras de la zona de Itacurubí de la Cordillera y de Natalicio Talavera, que consistió en brindarles las herramientas necesarias para que formen su propia cooperativa de trabajo considerando sus potencialidades y las actividades que desarrollan en el caso de las artesanas, toda una comunidad dedicada al tejido de prendas tradicionales, las mismas recibieron la entrega de capital semilla para consolidar la conformación de sus comités de artesanas y dando cumplimiento al objetivo del proyecto "Promover el surgimiento y consolidación de nuevos tipos de cooperativas como alternativas de desarrollo" y como principal acción del Plan Operativo 2014 de la CONPACOOP. El proyecto buscó la creación de nuevas formas de asociación basados en el trabajo y en la explotación de habilidades para el mejoramiento del bienestar de las personas beneficiadas y su comunidad. Actualmente, dicho proyecto no ha podido concretar la creación de la cooperativa de trabajo.

El presente trabajo tiene como objetivo recopilar la experiencia de asociatividad en mujeres tejedoras paraguayas en el distrito de Yataity, Departamento de Guaira; considerada cuna del ao po'i. 


\section{METODOLOGÍA}

La investigación se realizó en año 2015 a través de un análisis documentalbibliográfico, consultando a varios documentos de organismos nacionales e internacionales, como la Organización de las Naciones Unidas, el Consejo Latinoamericano de Ciencias Sociales, el Programa de las Naciones para el Desarrollo. Además, se recopilaron datos estadísticos de la Dirección General de Estadística, Encuestas y Censos y el Instituto Paraguayo de Artesanía.

Como trabajo de campo, se ha realizado una entrevista a profundidad a la representante del Instituto Paraguayo de Artesanía (IPA) de Yataity y a la Presidenta de la Cooperativa Multiactiva de Producción Artesenal Ao Poí Servicios Yataity Ltda.

Además se recurrió a la aplicación de un grupo focal con 12 miembros de la Red de Mujeres Artesanas de Ao Pói, siendo considerada como la más representativa no solo por sus años de presencia ininterrumpida, sino por nuclear a 3 comités de artesanas de Yataity.

Algunas preguntas que se han utilizado como disparadoras al grupo fueron; motivo por el cual decidieron tejer, si tejer es una actividad principal o secundaria, algunas ventajas o desventajas del trabajo, cual es la manera en la que comercializan los productos, si esto da sustento a las familias y como es el mercado para la venta.

\section{RESULTADOS}

Para las artesanas del Ao Po'i, la historia de dicha artesanía se remonta a tiempos en que fueron sus propias bisabuelas, las que iniciaron el armado de la tela, las más de 70 variedades de bordados y la confección de prendas. El conocimiento del mismo, fue trasmitido de generación en generación, de madres a hijas por lo general, la forma particular del bordado ha quedado en la memoria de cada una, ya que no existen registros ni documentos de los diversos tipos y variedades ni quien los haya creado. Cabe destacar, que cada artesana con los años, le fue dando su impronta personal, mejorando y creando el bordado en Ao Po'i.

De acuerdo a las artesanas entrevistadas, la comercialización de las prendas en ao po’i data de más de 40 años, y está no ha sido fácil, por la realidad con la 
que tropiezan, que es su poca o casi nula valoración, indicando que el público por lo general no dimensiona el trabajo que llevo hacer cada prenda, el esfuerzo que cada una imprime en su confección y sobre todo el agregado de creatividad, ya que es un arte.

Tejer Ao Po’i es hacerlo, no a tiempo completo, aproximadamente el 90\% de las mujeres que se dedican a este oficio, son amas de casa, y van haciendo en sus ratos libres mientras no atiendan los quehaceres del hogar ni el cuidado de sus hijos. Por lo tanto, el horario que las mismas dedican a la confección es por las tardes, compaginando entre sus quehaceres diarios. Además depende de las condiciones fisicas de las tejedoras para dedicarse largas horas a realizar esta actividad. El lugar de trabajo de las artesanas, no es un inconveniente, porque generalmente lo hacen en sus propias casas. Actualmente, el Instituto Paraguayo de Artesanía, tiene registrada a más de 700 artesanas, activas, en la ciudad de Yataity.

De acuerdo a la aplicación del método de grupo focal, a mujeres artesanas de la ciudad de Yataity, estas coincidieron que la labor de las mismas, se refuerza más a través de la asociatividad, ya que el mismo es rubro donde el individualismo y la labor en solitario no próspera por múltiples razones las cuales se detallan a continuación:

\section{Producción:}

Una artesana por si sola podría bordar y confeccionar productos en Ao Po'i en un número reducido. A modo de ejemplo, el bordador de un mantel de aproximadamente tres metros llevaría a una sola artesana hacerlo en 2 a 3 años, mientras que si trabajan entre varias tardarian no más de 6 meses en terminarlo.

Para iniciar un pedido, generalmente cada tejedora compra sus insumos con su propio capital, en caso que no lo tenga, compra endeudándose hasta obtener el dinero y pagar el préstamo realizado.

Se da también la situación de que, se reciben los pedidos, pero muchas veces no todas las artesanas pueden cumplir con un pedido y en las fechas solicitadas. Por lo tanto, existe siempre un grupo reducido que trabaja incansablemente para hacer frente a dicha demanda.

\section{Canales de Ventas:}


Para comercializar la mismas necesitan de un grupo, porque trabajando en forma individual accederian a un reducido mercado, mientras que si lo hacen como miembro de una entidad, tendrian mayores posibilidades de asegurar la venta de sus productos y por ende obtener mejores retornos económicos.

En el caso de la Cooperativa Ao Po'i, los pedidos se reciben en dicha entidad y la misma difunde entre sus asociadas para hacer a la demanda. Además les brinda la posibilidad de que las mismas puedan vender sus productos a la propia entidad, percibiendo un monto por encima de los grandes acopiadores, o empresarios que se dedican a la venta de prendas artesanales. Por lo tanto, a las mismas se les paga por el bordado que realizan, luego la propia entidad se encarga de poner en condiciones para la venta, realizando el control de calidad, acorde a las exigencias del cliente. En el caso de la Red de Mujeres Artesanas de Yataity, las mismas confeccionan las prendas y lo venden en su propio local.

El Instituto Paraguayo de Artesanía (IPA) apoya a las asociaciones de artesanas en las ferias internacionales, asistiéndole con el pasaje, viático, transporte si es regional e internacional. En las expo y ferias, proveen de la instalación necesaria para la exhibición de los productos (toldos, mesas y sillas). En este sentido, el IPA les otorga un carnet identificador a cada artesana registrada, para ello, la artesana debe demostrar a través de un muestrario su trabajo, con el cual, la institución verifica que la misma se dedica a dicho oficio.

En el caso de exportación de productos, anteriormente el IPA ofrecía a las artesanas este servicio, pero actualmente es la Red de Inversiones y Exportaciones (Rediex) dependiente del Ministerio de Industria y Comercio (MIC), los que facilitan el envío de los productos al exterior, de lo contrario sería muy costoso para las artesanas.

Cuando se trata, sobre todo de artesanas independientes, las mismas trabajan para diseñadores o modistas de alta costura, los cuales compran solo telas bordadas, como complemento de la prenda principal que confeccionan.

Con miras a ampliar el mercado, se ha tratado de innovar e incursionando como complemento para los productos de blanquería, pero por el costo, estos no progresaron. Los creadores de otros productos, ven a la confección del Ao Po’i como un producto caro que encarece el producto final. Existen productos cuyas ventas son más rápidas y otras que se mantienen en stock por un largo periodo, y este es el caso de los manteles, que debido al alto precio no se consideran como productos 
de "salida rápida" en el mercado local, por lo que muchas veces el precio de venta no compensa con todo el esfuerzo realizado. Los productos, actualmente más vendidos son las blusas para damas y camisas para caballeros, además de los denominados "apliques" que sirven de complemento para la confección de prendas de vestir a base de otro tipo de tela.

\section{Acceso a Créditos y otros beneficios:}

Por reglamento y objetivos que persigue el IPA, las tejedoras deben de estar asociadas en comités, asociaciones o cooperativas. Estas obtienen su certificación por parte del IPA como empresa artesanal, obteniendo beneficios con el IVA, esto se debe a un Convenio entre el IPA y el Ministerio de Hacienda, por lo que, cada artesana certificada y con carnet tiene exonerado el pago del IVA para realizar sus transacciones de compra y venta.

Entre las ventajas de la asociación, es que las mismas acceden a más ayuda, capacitación y tienen más posibilidades de recibir apoyo técnico o crediticio. Por lo que generalmente se les solicita estar asociadas.

Actualmente, a través de un proyecto denominado "Paraguay Inclusivo" del Ministerio de Agricultura y Ganadería en conjunto con la Gobernación de Guairá, ha beneficiado a más de 100 mujeres artesanas asociadas. Las mismas pudieron acceder a los beneficios de este programa gracias a que poseian cuenta bancaria, personería jurídica, estatuto, entre otros; que de lo contrario les sería difícil acceder.

Las desventajas del asociativismo, es que muchas veces, es dificil trabajar en grupo, porque existe mucha competencia en cuanto a la calidad del producto, el mercado tiene sus exigencias y muchos grupos de artesanos no cumplen con los estándares de calidad, lo cual dificulta poder acceder a un mayor mercado.

Otra institución que otorga crédito a las artesanas es el Crédito Agrícola de Habilitación $(\mathrm{CAH})$, a través de un programa para actividades no agrícolas de mujeres campesinas.

\section{DISCUSIÓN}

El Ao Po'i, un trabajo temporal, mientras las tejedoras no obtienen pedidos, las mismas realizan trabajos para el puesto de venta, a través de la confección de 
prendas para reponer stock o mejorar las variedades y surtidos. Y por otro lado, las que no están asociadas se dedican al bordado y trabajan para un gran acopiador y cobran por ese único trabajo. Las independientes venden sus trabajos a personas que poseen negocios, quienes compran y pagan en efectivo por la mercancía, lo cual es muy beneficioso para las artesanas.

En los últimos años, las ventas han disminuido bastante, lo que ha hecho que las artesanas se dediquen a actividades que complementen los ingresos del hogar, tales como; ferias de comidas, actividades en el rubro de panadería y confitería, cría de aves menores y cultivos agrícolas.

Otras cuestiones a tener en cuenta, es que el Ao Po’i es un producto que no se vende en los meses de invierno, por las características de la tela, la misma es para clima tropical, por ello, las ventas se acentúan en los meses de calor. Por lo tanto, para hacer frente a la demanda que pueda surgir en los meses de verano, muchas veces planificar y preparar productos para esa temporada es dificil, ya que es un producto muy costoso para tenerlo en stock, por el hecho de que la subsistencia del hogar para la mayoria de las artesanas depende directamente de sus ventas diarias.

Por otro lado, la exportación es considerada una gran oportunidad para las mismas. La proyección a buscar nuevos mercados y ampliar el ya existente, es uno de los objetivos de promoción de las exportaciones del Ministerio de Industria y Comercio de Paraguay, para ello, las entrevistadas comentaron que se realizaron reuniones informativas en la oficina Regional del Ministerio de Industria, con el objetivo de poner a conocimiento de las artesanas el proyecto de crear una "marca producto" con miras a la exportación, lo cual implica montar un taller para las confeccionistas y trabajar en conjunto con las bordadoras.

De acuerdo a lo mencionado por la Directora Regional del IPA, el crear una sola marca que identifique y represente al producto de la ciudad tiene sus dificultades, ya que esto implicaria cambio en el sistema de producción, por el hecho de que el Ao Po'i es un producto artesanal, hecho a mano. Existen falencias en la confección, por el hecho de que no existe uniformidad en la misma, el ponerse de acuerdo y seguir las reglas, es dificil entre la artesanas, ya que cada una sigue su propio estilo. En este sentido, ha mencionado la experiencia que han tenido al realizar una producción masiva de las mercaderías años atrás, argumentando que; "Una prenda hecha a mano no puede ser tan perfecta como otra hecha a máquina", por lo que es dificil trabajar de manera industrial. 
Para fortalecer la producción de Ao Poí, años atrás, el IPA había adquirido máquinas profesionales para el corte de tela, pero el Ao Po’i tiene la característica que no puede ser confeccionada en forma masiva, sino en forma individual.

Otro factor negativo no menor para la exportación, tiene que ver con la logística, se debe de llenar un contenedor marítimo, o de lo contrario no puede llevarse la mercancía al exterior. Por vía aérea es bastante caro, dado el pago por peso y volumen.

Además, para exportar mínimamente se requieren de una cantidad aproximada de 5.000 prendas, y muchas veces las empresas dedicadas a la misma, pagan un precio muy por debajo de su costo de producción, lo que desmotiva a las propias artesanas producir en grandes cantidades para este fin.

Con respecto a las capacitaciones; para aprender a tejer el Ao Po'i y preservar el legado de los ancestros, se imparten clases de telar rústico en las instituciones educativas, tanto de las zonas urbanas como de las rurales de la ciudad de Yataity. Las tejedoras por su lado, también han recibido capacitaciones ya sea para la mejora en la producción como así también para conocer sobre técnicas de marketing, planificación, costo de producción y ventas, entre otros.

Uno de los factores con los cuales tropiezan las artesanas, es que, por más que se hayan hecho vastas capacitaciones, las mismas, siguen una linea rígida de no innovar o seguir una línea estándar y de no uniformidad. Las entrevistadas afirman que: "La persona que tienen disciplina y responsabilidad es la que progresa. Pero la mayoría son conformistas, también es un factor negativo cultural y afecta a la producción".

Otra situación que se da, es que las nuevas generaciones han perdido el interés de dedicarse a este oficio, por el hecho de ser poco rentable, por lo tanto, se dedican a otras profesiones o aprenden otros oficios.

En cuanto a los costos de producción, se han realizado capacitaciones con el objetivo de que las artesanas adquieran destrezas y habilidades de negociación, cálculo de costos y precio de venta, hubieron cursos que tuvieron una duración de aproximadamente 6 meses en la Cooperativa Ao Po'i, la Red de Mujeres Artesanas también recibieron capacitación a través de un proyecto de la Organización sin Fines de Lucro denominado Eco Global. Estos cursos tuvieron como objetivo formarlas como microempresarias, pero la realidad con que muchas de ellas se 
encuentran es que, la venta de sus productos se rige muchas veces por la disposición a pagar del comprador, que generalmente es menor al precio de venta real.

La producción lleva tiempo y esfuerzo por parte de las mismas, por lo general una artesana puede llegar a bordar hasta 2 camisas en una semana, lo que implica una remuneración bastante baja. Haciendo que está actividad no sea considerada como principal, sino como actividad de ingreso alternativo $y$ siendo un complemento a los ingresos de sus cónyuges.

Una de las principales criticas que las artesanas destacan, es que, muchos proyectos de organismos del gobierno nacional llevan ilusiones que acaban en la nada, son temporales, no teniendo en la mayoria de los casos monitoreo de parte de las autoridades ni continuidad. La información muchas veces resulta asimétrica en cuanto a propuestas de participación en ferias, lo que hace que la información solo llegue a ciertos grupos, no se socialice, por ende, solo beneficia a unos y excluye a otros.

Finalmente, la asociatividad implica compromiso y una de las razones por las que no se asocian a una entidad es porque no se encuentran en condiciones de asumir las exigencias en cuanto a aporte monetario, apoyo en el local de ventas, realización y cumplimiento con pedidos en tiempo y forma, remuneraciones exiguas, discrepancias y otros motivos personales.

\section{REFERENCIAS}

Beauvoir, S. de, López Pardina, T., \& Martorell, A. (2005). El segundo sexo. Madrid: Cátedra.

Berdegue, J., Olfert, M. R., Partridge, M., Escobal, J., Jara, B., \& Modrego, F. (2014). Places for Place-Based Policy. Development Policy Review, 32(1), 5-32.

Bremer, M. (2016). Tres diferentes sistemas económicos. Coordinación Nacional de Pastoral Indigena (CONAPI). Recuperado a partir de http://www.conapi.org.py/documentos/35/3_diferentes_sistemas_economico s.pdf

Carosini, L., \& Achinelli, M. (2015). Las Cooperativas de Trabajo en América Latina. (M. Shujman, Ed.). Argentina: Del Revés. Recuperado a partir de https://www.academia.edu/16516469/Las_Cooperativas_de_Trabajo_en_Am $\%$ C3\%A9rica_Latina 
CONPACOOP. (2012). Censo Nacional Cooperativo. Confederación Paraguaya de Cooperativas Limitada (CONPACOOP). Recuperado a partir de http://www.conpacoop.coop.py/observatoriocooperativo.php

Echauri, C., \& Serafini, V. (2011). Igualdad entre hombres y mujeres en Paraguay: la necesaria conciliación entre familia y trabajo. Santiago: OIT.

Espinosa Miñoso, Y., Gómez Correal, D. M., \& Ochoa Muñoz, K. (Eds.). (2014). Tejiendo de otro modo: feminismo, epistemología y apuestas descoloniales en Abya Yala. Popayán, Colombia: Editorial Universidad del Cauca.

Forstner, K. (2013). La artesanía como estrategia de desarrollo rural: el caso de los grupos de artesanas en la región de Puno (Perú). Cuadernos de Desarrollo Rural, 10(72), 141-158.

Girón, A., \& Vargas, V. (Eds.). (2009). Género y globalización (1. ed). Buenos Aires: CLACSO.

Martinez, J. C. (2002). Las cooperativas en América Latina: visión histórica general y comentario de algunos países tipo. CIRIEC - España. Revista de economía pública, social y cooperativa, (43), 145-172.

Moser, C. (1992). Adjustment From Below: Low-Income Women, Time and the Triple Role in Guayaquil, Ecuador, 10.

Norverto, L. (2014). "Trabajo" y "proyecto propio": aportes teórico-metodológicos de género y microemprendimientos de mujeres. "Work" and "Own Project": Theoretical-Methodological Contributions Based on Gender and Women Microentrepreneurs., 9(1), 43-52.

Potthast, B. (1996). "Paraíso de Mahoma" ,o, "País de las mujeres"?: el rol de la familia en la sociedad paraguaya del siglo XIX. Instituto Cultural ParaguayoAlemán.

Potthast, B. (2006). Algo más que heroínas. Varias roles y memorias femeninas de la Guerra de la triple alianza. Diálogos - Revista do Departamento de História e do Programa de Pós-Graduação em História (Brasil), 10(1), 89-104.

Ranaboldo, C., \& Leiva, F. (2013, junio). La valorización de los activos culturales: ¿Estratgias innovadoras para el empoderamiento de las mujeres rurales jóvenes? Institutos de Estudios Peruanos. Recuperado a partir de http://www.rimisp.org/wp-

content/files_mf/1377802149lavalorizaciondelosactivosculturalesestrategiasin novadorasparaelempoderamientodelasmujeresruralesjovenes.pdf 
Readon, B. (2001). Education for a Culture of Peace in a Gender Perspective. International Review of Education, 49(3), 395-399. http://doi.org/10.1023/A:1025351804453

Sen, A., \& Rabasco, E. (2000). Desarrollo y libertad. México; Barcelona: Planeta.

Serafini Geoghegan, V. (2008). La liberalización económica en Paraguay y su efecto sobre las mujeres (1. ed). Buenos Aires: CLACSO.

UNESCO. (2009). Marco de Estadísticas Culturales de la UNESCO 2009. Recuperado el 31 de mayo de 2016, a partir de http://www.uis.unesco.org/culture/Documents/framework-culturalstatistics-culture-2009-spa.pdf

Villamil Peña, M., \& Quevedo, R. (1987). Silvia. Paraguay: Criterio. 